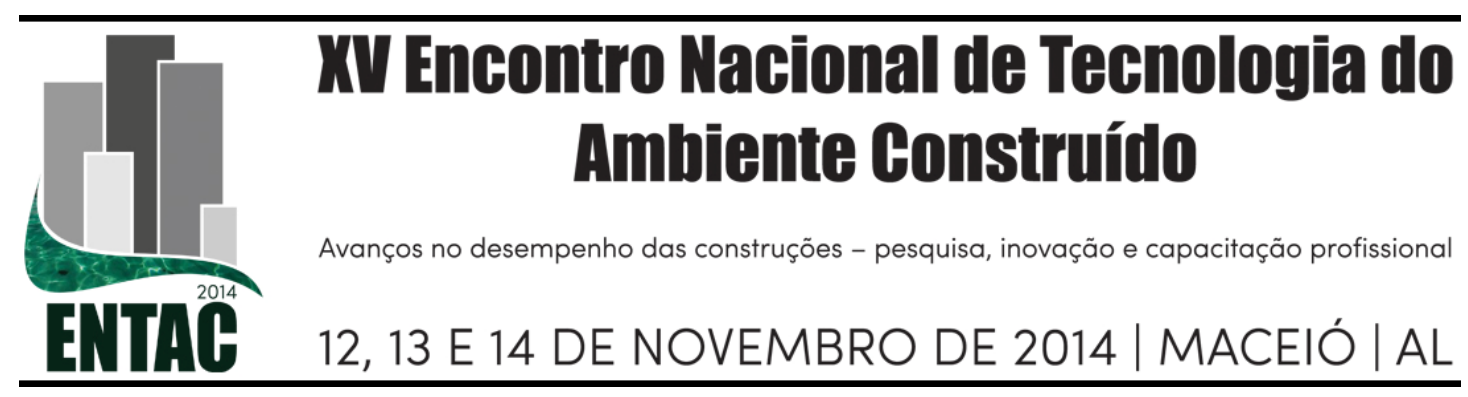

\title{
SIMULAÇÃO COMPUTACIONAL DO ESCOAMENTO EM SISTEMAS PREDIAIS DE ESGOTO SANITÁRIO POR MEIO DE UM MÉTODO DE PARTÍCULAS
}

\author{
CHENG, Liang-Yee (1); OLIVEIRA, Lúcia Helena (2); \\ FAVERO, Eric Henrique (3) ; OLIVEIRA, Igor Bernardes (4); \\ GONÇALVES, Orestes Marraccini (5)
}

(1) PCC/EP/USP, (11)3091-5462, e-mail: cheng.yee @ poli.usp.br, (2) PCC/EP/USP, e-mail:

lucia.oliveira@poli.usp.br, (3) PCC/EP/USP, e-mail: eric.favero@usp.br, (4) PCC/EP/USP, email: igor.bernardesoliveira@usp.br, (5) PCC/EP/USP, e-mail:

orestes.goncalves@poli.usp.br

\begin{abstract}
RESUMO
O objetivo desta pesquisa é prover uma ferramenta computacional eficaz para investigar o impacto de redução da vazão em sistemas prediais de esgoto sanitário, devido ao uso racional da água. Para isso, um método de partículas para simular a dinâmica de fluídos é utilizado para modelar o complexo escoamento transitório com superfícies livres em tubulações horizontais. Como caso de estudo, escoamentos transitórios em um sistema de esgoto sanitário de um banheiro com uma bacia sanitária, um chuveiro e um lavatório, localizado no segundo andar foi considerado. Os resultados mostram boa concordância com algumas medições experimentais e permitem uma análise detalhada do processo hidrodinâmico.
\end{abstract}

Palavras-chave: Sistemas prediais de esgoto sanitário, simulação computacional, método de partículas.

\begin{abstract}
The aim of the present research is to provide an effective computational tool to investigate the effects of the reduction of flow rate caused by water conservation practices on the performance of the building drainage systems. In order to model the complex transient free surface flow inside the drainage system, a particle-based computational fluid dynamic technique is adopted. As case of study, the drainage from a bathroom with a water closet, a shower and a wash basin located at the second floor is considered. The results of the simulation are given and they show a good agreement with the ones obtained by measurement and allow a detailed analysis of the hydrodynamic process.
\end{abstract}

Keywords: Building drainage system, computer simulation, particle method.

\section{INTRODUÇÃ̃O}

Nos últimos anos, as práticas de redução de consumo de água têm contribuído para a redução da vazão nos sistemas prediais de esgoto sanitário. Tendo em vista o impacto da redução da vazão sobre a capacidade de transporte de dejetos e de autolimpeza do sistema, torna-se imprescindível a avaliação da redução da vazão no sistema tanto em projetos novos como em edificações existentes (McDOUGALL e SWAFFIELD, 2003; LEE, 2006; CHENG et al, 2013). Algumas ferramentas ou estudos embasados nas técnicas de simulação computacional foram reportados (JACK e SWAFFIELD, 2009). No entanto, a modelagem do completo processo hidrodinâmico continua sendo um desafio. Nestes contexto, o objetivo deste trabalho é prover uma ferramenta 
computacional eficaz para simular o complexo escoamento transitório com superfícies livres dentro de tubulações horizontais e realizar uma investigação numéricocomputacional do desempenho de um sistema predial de esgoto sanitário. Para isso, uma abordagem inovadora da Dinâmica dos Fluídos Computacional é adotada. A abordagem é baseada no método Moving Particle Semi-Implicit (MPS), que utiliza uma descrição completamente lagrangeana e discretiza o domínio computacional em partículas móveis. Por ser um método que não utiliza malhas de cálculo, o método é extremamente eficaz para simular fenômenos hidrodinâmicos que envolvem superfícies livres, fragmentação de junção de líquidos, grandes deformações, grandes deslocamentos e corpos de geometrias complexas, além dos problemas de multicorpo, multifase e multifísicos.

A abordagem já foi aplicada para estudar os efeitos da redução do diâmetro e da declividade num ramal horizontal (CHENG et al., 2012), assim como o estudo do escoamento numa junção de $45^{\circ}$ e num ramal de esgoto (CHENG et al., 2013). Neste trabalho, especificamente, foram realizadas modelagem e simulação computacional dos escoamentos transitórios em um banheiro de edifício residencial considerando uma bacia sanitária, um chuveiro e um lavatório localizado no segundo andar. Perfis típicos das vazões dos aparelhos sanitários foram utilizados na modelagem, que engloba a junção entre os dois ramais de esgoto e este com o tubo de queda, assim como o escoamento em torno da ligação do tubo de queda com o subcoletor, que é feita por meio de dois joelhos de $45^{\circ}$. Resultados e discussões sobre os diferentes padrões de ondas formados e as séries temporais de vazão em diversas seções são apresentados. Por simplicidade, os efeitos de ar no interior das tubulações são desprezados, assim como o fenômeno de transporte de sólidos, que serão alvos das próximas etapas do trabalho.

A seguir, é apresentada uma breve descrição do método numérico adotado, os casos de estudo e a análise dos resultados obtidos.

\section{MÉTODO NUMÉRICO}

\subsection{Equações governantes}

As equações governantes do escoamento incompressível e viscoso do problema a ser analisado neste estudo são equação de continuidade:

$$
\frac{D \rho}{D t}=-\rho(\nabla \cdot \vec{u})=0
$$

e equação de conservação de quantidade de movimento:

$$
\frac{D \vec{u}}{D t}=-\frac{1}{\rho} \nabla P+v \nabla^{2} \vec{u}+\vec{g}
$$

onde, $\rho=$ densidade;

$\vec{u} \quad=$ velocidade;

$P \quad=$ pressão;

$v \quad=$ viscosidade cinemática;

$g \quad=$ aceleração gravitacional.

\subsection{Operadores algébricos}

Originalmente proposto por Koshizuka e Oka (1996) para simular o escoamento incompressível com superfície livre, o método numérico do qual se baseia este trabalho 
é o Moving Particle Semi-implicit (MPS). O MPS é um método sem malha que utiliza descrição Lagrangeana, no qual o domínio espacial é discretizado em partículas. $\mathrm{O}$ método modela o escoamento na escala macroscópica, como meios contínuos, e resolve as equações (1) e (2) substituindo os operadores diferenciais por operadores algébricos definidos a partir de um modelo de interação entre partículas baseado na função peso dada por:

$$
w(r)=\left\{\begin{array}{cc}
\frac{r_{e}}{r}-1, & \left(r<r_{e}\right) \\
0, & \left(r>r_{e}\right)
\end{array}\right.
$$

onde, $r=$ distância entre duas partículas;

$r_{e} \quad=$ o raio efetivo.

O raio efetivo $r_{e}$ limita a região onde a interação entre as partículas ocorre.

O número de densidade de partícula ( $p n d$ ) é definido como a soma do peso de todas as partículas $j$ vizinhas, conforme a equação (4):

$$
\langle p n d\rangle_{i}=\sum_{i \neq j} w\left(\left|\vec{r}_{j}-\vec{r}_{i}\right|_{i}\right)
$$

onde, $\vec{r}_{i} \quad=$ vetor posição da partícula $i$;

$\vec{r}_{j} \quad=$ vetor posição das partículas $j$, vizinhas à $i$.

$\mathrm{Na}$ equação (4) e nas subsequentes, \langle\rangle indica operadores algébricos, e os índices subscritos $i$ e $j$ indicam, respectivamente, a partícula considerada e suas vizinhas.

O número de densidade de partícula ( pnd ) é proporcional à densidade do fluido e é um parâmetro importante do método, pois é utilizado como referência para assegurar a incompressibilidade do escoamento. Ou seja, num escoamento incompressível, o pnd de todas as partículas deve ser constante e igual ao $p n d^{0}$, que é seu valor inicial.

A Figura 1 mostra o exemplo de um domínio modelado por partículas lagrangeanas e os principais tipos de partículas utilizados neste estudo, assim como o raio efetivo $r_{e}$.

Dada uma função escalar $\phi$, o vetor gradiente e o Laplaciano da função numa partícula $i$ podem ser determinados por meio das equações (5) e (6), respectivamente:

$$
\begin{gathered}
\langle\nabla \phi\rangle_{i}=\frac{d}{p n d^{0}} \sum_{i \neq j}\left[\frac{\left(\phi_{j}-\phi_{i}\right)}{\left|\vec{r}_{j}-\vec{r}_{i}\right|^{2}}\left(\vec{r}_{j}-\vec{r}_{i}\right) w\left(\left|\vec{r}_{j}-\vec{r}_{i}\right|\right)\right] \\
\left\langle\nabla^{2} \phi\right\rangle_{i}=\frac{2 d}{p n d^{0} \lambda} \sum_{i \neq j}\left[\left(\phi_{j}-\phi_{i}\right) w\left(\left|\vec{r}_{j}-\vec{r}_{i}\right|\right)\right]
\end{gathered}
$$

onde, $d \quad=$ número das dimensões espaciais;

pnd $^{0}=$ valor inicial do número de densidade de partícula;

$\lambda=$ parâmetro de ajuste da taxa de difusão.

Nas equações (5) e (6), $d$ é igual 2 e 3 para problemas bidimensionais e tridimensionais, respectivamente. $\mathrm{O}$ parâmetro $\lambda$ é calculado por: 


$$
\lambda=\frac{\sum_{i \neq j}\left|\vec{r}_{j}-\vec{r}_{i}\right|^{2} w\left(\vec{r}_{j}-\vec{r}_{i} \mid\right)}{\sum_{i \neq j} w\left(\left|\vec{r}_{j}-\vec{r}_{i}\right|\right)}
$$

Figura 1 - A modelagem do domínio fluido, superfície livre e paredes por partículas e o raio efetivo $\left(r_{e}\right)$ de uma partícula $i$ e suas vizinhas $j$.

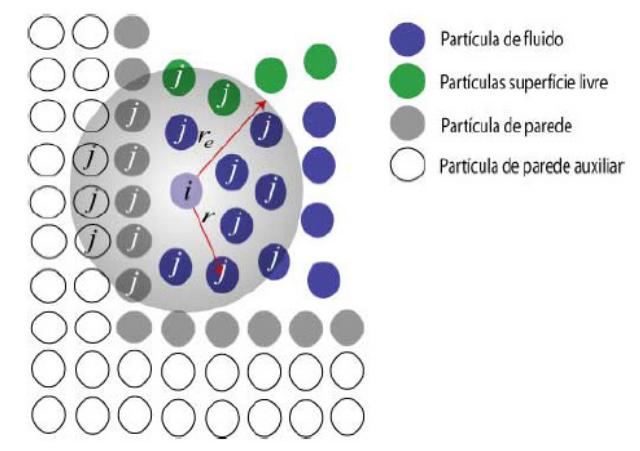

\subsection{Condições de contorno}

Conforme mostram as partículas de cor verde na Figura 1, quando uma partícula está na superfície livre, suas partículas vizinhas não preenchem totalmente sua região de vizinhança, e o número de densidade de partícula é menor que $p n d^{0}$. Sendo assim, o valor $\beta \cdot p n d^{0}$ pode ser adotado para identificar se uma partícula é ou não de superfície livre. Ou seja, uma partícula $i$ é considerada como partícula de superfície livre quando:

$$
\langle p n d\rangle_{i}<\beta \cdot p n d^{0}
$$

Aplicando a condição dinâmica da superfície livre, a pressão de todas as partículas que satisfazem este critério é imposta como zero. De acordo com Koshizuka e Oka (1996), o valor de $\beta$ adotado neste trabalho é 0,85 .

Tradicionalmente, três fileiras de diferentes tipos de partículas são usadas para descrever a geometria de uma parede rígida no MPS. A pressão é calculada nas partículas da primeira fileira, em contado com fluido, denominadas de partículas de parede e representadas na cor cinza na Figura 1. As outras duas fileiras de partículas que não estão em contato com o fluido são formados por partículas de parede auxiliar, ou partículas dummy, que são utilizadas para garantir o cálculo correto do pnd das partículas de parede. As partículas dummy são representadas em cor branca e não é necessário calcular a pressão delas.

A fim de modelar o afluxo de esgoto no sistema, a condição de contorno de afluxo é aplicada na seção à montante do trecho em estudo. Do lado externo da seção do afluxo, uma parede é deslocada em direção normal à seção com velocidade definida pela vazão instantânea do escoamento. Quando as partículas de parede cruzam completamente a seção, elas são convertidas em partículas de fluido. Ao mesmo tempo, as partículas dummy adjacentes às partículas de parede são convertidas em partículas de parede, e novas partículas dummy são adicionadas no lado à montante da parede para assegurar que existem sempre duas camadas de partículas dummy ao lado das partículas de parede. No caso do escoamento anular do tubo de queda, ao invés da seção transversal do escoamento, a condição de afluxo é aplicada apenas na forma anular da seção. 


\subsection{Algoritmo}

O método MPS adota um algoritmo semi-implícito. Exceto o termo de gradiente de pressão, os termos do lado direito da Eq. (2) são calculados explicitamente para estimar velocidade e posição das partículas. Em seguida, a equação Poisson de pressão, dada pela Eq. (9), é resolvida de forma implícita para o instante $(t+\Delta t)$ :

$$
\left\langle\nabla^{2} P\right\rangle_{i}^{t+\Delta t}=-\frac{\rho}{\Delta t^{2}} \frac{p n d_{i}^{*}-p n d^{0}}{p n d^{0}}
$$

onde, $\quad p n d^{*}=$ o número de densidade de partícula calculado explicitamente.

Conforme mencionado no item 2.3, o número de densidade de partícula calculado usando a posição estimada das partículas na parte explícita do algoritmo, pnd ${ }^{*}$, é mantido igual a $p n d^{0}$ para assegurar a condição de incompressibilidade do escoamento.

O termo do lado esquerdo da Eq. (9) pode ser discretizado usando o modelo do Laplaciano, isto é, a Eq. (6), constituindo assim, um sistema de equações. Uma vez obtido o valor de pressão em todas as partículas, realiza-se a correção das velocidades e posições. Para o presente estudo, $r_{e}$ foi estabelecido como 2,1.

\section{ESTUDO DE CASO}

O sistema de esgoto sanitário de um banheiro com bacia sanitária, lavatório e chuveiro e localizado no segundo andar é considerado neste estudo. A Figura 2 mostra a configuração e as dimensões do sistema. Apesar da aparente simplicidade do sistema, o complexo escoamento com superfície livre em duas singularidades típicas dos sistemas prediais de esgoto sanitário são levadas em conta:

- Escoamento transitório numa junção de $45^{\circ}$.

- Escoamento transitório e anular na transição tubo de queda - subcoletor.

Figura 2 - Esquema do sistema estudado com as dimensões e os focos da análise (Casos A e B)

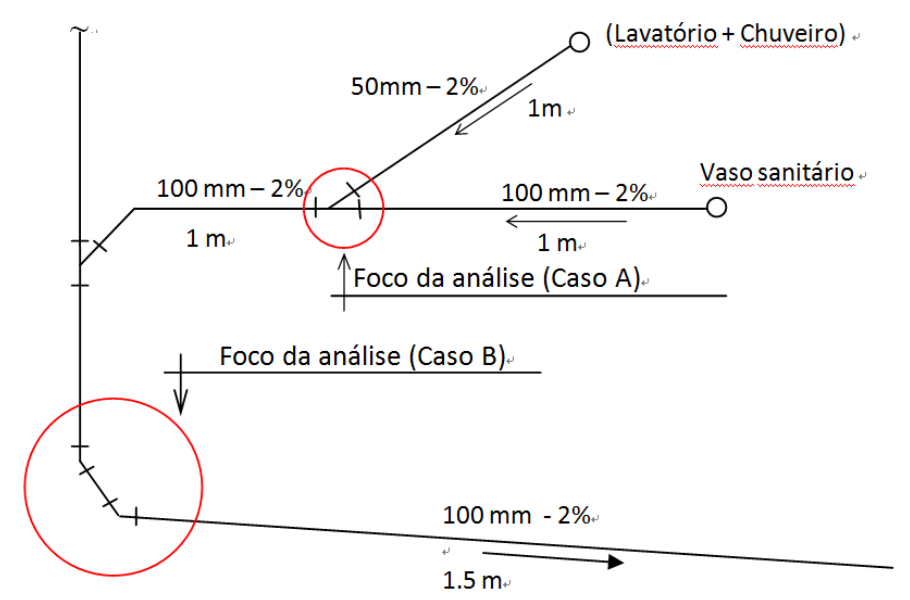

A fim de simplificar a modelagem e reduzir o custo do processamento, a investigação foi realizada separando o sistema em duas partes:

- Caso A: Escoamento transitório numa junção recebendo a vazão constante da caixa sifonada (lavatório e chuveiro) e uma vazão da descarga da bacia sanitária. 
- Caso B: Escoamento transitório na ligação tubo de queda - subcoletor, realizada por meio de dois joelhos de $45^{\circ}$.

A modelagem numérica foi feita usando a distância de partículas de $2 \mathrm{~mm}$, passo de tempo de $10^{-4}$ s e $20 \mathrm{~s}$ de tempo de simulação para o Caso A. Para o Caso B, os valores são, respectivamente, $1 \mathrm{~mm}, 5 \times 10^{-5} \mathrm{~s}$ e $10 \mathrm{~s}$. Uma resolução mais alta foi usada no Caso B para modelar melhor o fino filme de escoamento anular que desce pelo tubo de queda. Sendo assim, os modelos dos casos A e B envolvem, respectivamente, em torno de 2,3 e 5 milhões de partículas.

\section{RESULTADOS E DISCUSSÕES}

\subsection{Caso A}

No caso A um ramal de esgoto de $100 \mathrm{~mm}$ de diâmetro, $2 \mathrm{~m}$ de comprimento e $2 \%$ de declividade recebe a descarga de uma bacia sanitária. No seu ponto médio, uma junção de $45^{\circ}$ recebe um ramal de esgoto de $50 \mathrm{~mm}$ de diâmetro e $1 \mathrm{~m}$ de comprimento com as vazões de um chuveiro e um lavatório. Os efluentes deste ramal são conduzido ao tubo de queda. Tendo em vista que o uso do chuveiro e do lavatório é realizado com vazão constante e que a duração do uso é muito mais longa que a duração da descarga da bacia sanitária, os escoamentos oriundos dos dois primeiros são considerados em regime permanente. Sendo assim, o escoamento constante de $0,35 \mathrm{~L} / \mathrm{s}(0,15 \mathrm{~L} / \mathrm{s}$ do lavatório e $0,20 \mathrm{~L} / \mathrm{s}$ do chuveiro) é imposto ao ramal de esgoto de $50 \mathrm{~mm}$ da caixa sifonada. A outra contribuição refere-se ao ramal de descarga da bacia sanitária com volume de descarga de 6 litros, cujo perfil simplificado de vazão de descarga é dado na Figura 3. Visando a validação da modelagem, o perfil simplificado foi obtido por meio do ajuste da função senoidal aos pontos de medidos experimentalmente.

Figura 3 - Perfil simplificado da descarga da bacia sanitária com volume de descarga de 6 litros

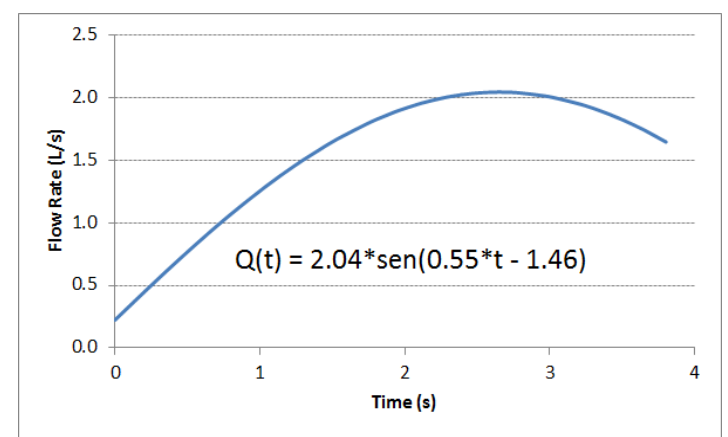

O escoamento no ramal de esgoto da caixa sifonada começa no $t=0,0 \mathrm{~s}$, e no instante $t=10,0 \mathrm{~s}$, quando o escoamento no sistema devido às vazões do chuveiro e lavatório já atingiram regime permanente, inicia a descarga da bacia sanitária. As quatro seções, S1, S2, S3 e S4, para o monitoramento da profundidade do escoamento e da vazão do Caso A estão posicionadas a $0,25,0,75,1,25$ e $1,75 \mathrm{~m}$ do ponto inicial do ramal de $100 \mathrm{~mm}$.

Os dois pontos críticos analisados são as seções S2 e S3 e ilustrados na Figura 4, que mostra a sequência de animação do escoamento no Caso A. Em cada instante, duas perspectivas são mostradas: no lado esquerdo a escala de cor da perspectiva indica a magnitude da velocidade. Dois anéis pretos ilustram a posição das seções S2 e S3. Na coluna da direita, a imagem mostra o processo de mistura onde a cor indica a origem do 
fluido. As partículas azul claro representam o efluente do chuveiro e do lavatório enquanto que as partículas azul escuro representam o efluente da bacia sanitária.

Figura 4-Caso A, velocidade (esquerda) e origem do fluxo (direita).
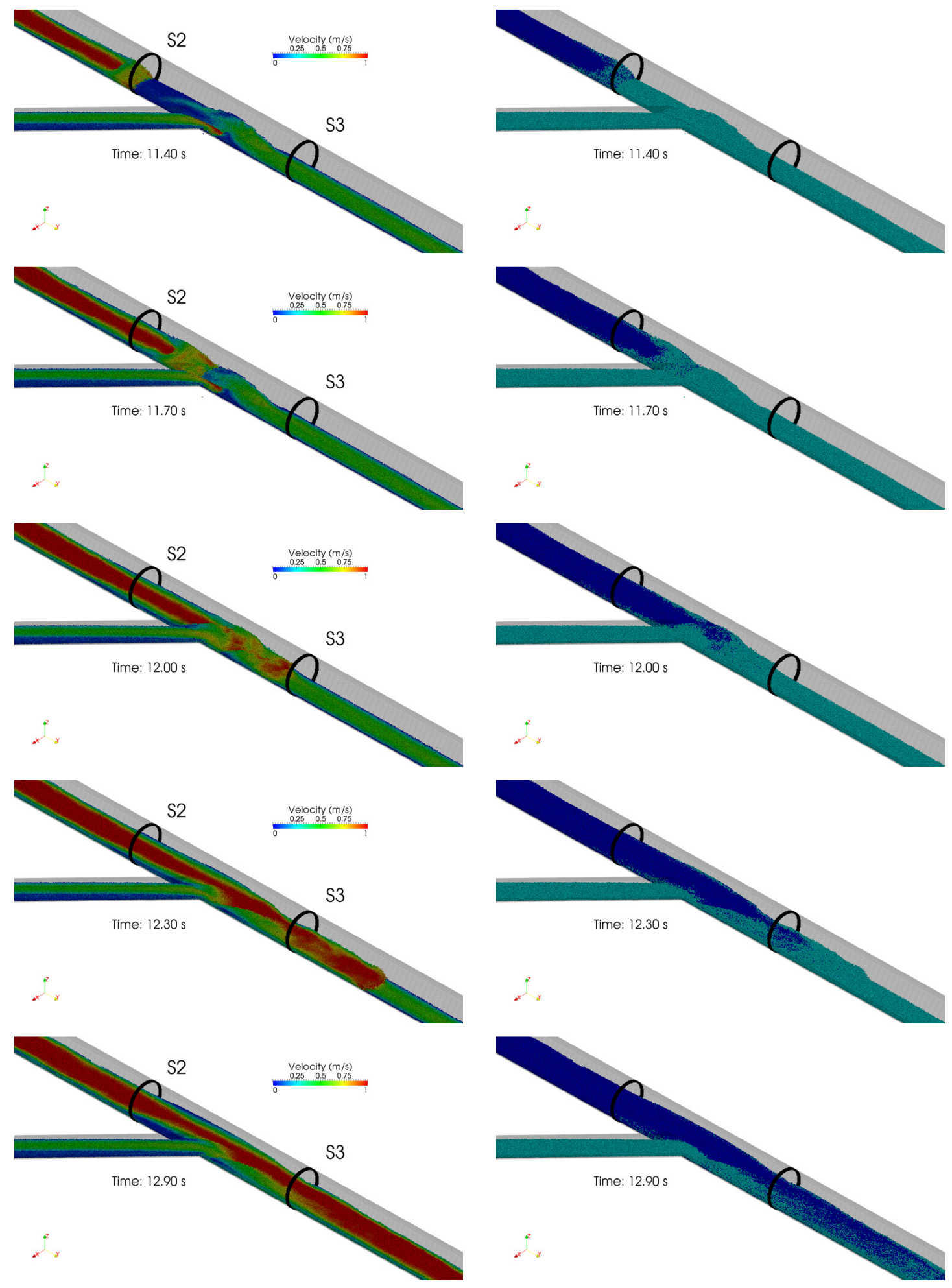

Inicialmente o efluente da caixa sifonada encontra-se em regime permanente, com uma parte do fluido atingindo a seção S2 à montante, enquanto que o fluxo total escoa na direção da seção à jusante S3. No instante $t=11,4 \mathrm{~s}$, a frente de onda devido a descarga da bacia sanitária atinge a seção $\mathrm{S} 2$. No $t=11,7 \mathrm{~s}$ a frente de onda chega à junção e 
encontra com o fluxo vindo do ramal de esgoto da caixa sifonada formando um padrão de escoamento complexo com ondas tridimensionais e pontos de estagnação próximos à junção. No instante $t=12,0 \mathrm{~s}$, a frente de onda cruza a junção e gera intensa mistura dos fluídos. Em seguida, juntamente com a passagem do pico da vazão da descarga, formase um escoamento assimétrico e com processo de mistura relativamente estável por cerca de 2 segundos, até o nível e a velocidade reduzir e retornar ao estado anterior à descarga da bacia sanitária.

As séries temporais de profundidade do escoamento da água na seção S3 obtidas experimentalmente e as calculadas numericamente são mostradas na Figura 5. A linha azul mostra os resultados da simulação, enquanto que os pontos marrons são obtidos por medições experimentais. A profundidade nas seções foram medidas com base nas imagens obtidas na filmagem. Tendo em vista a complexidade da geometria da superfície livre, foram utilizados os valores médios obtidos dos vídeos gravados de ambos os lados da tubulação de acrílico transparente. A Figura 5 mostra um aumento brusco da altura do escoamento em torno do $t=12,1 \mathrm{~s}$, que corresponde ao instante que a frente de onda atinge a S3. O nível eleva-se de uma forma mais gradual até por volta de $t=14,0 \mathrm{~s}$, e depois ocorre uma queda brusca seguida de uma redução gradual da altura do escoamento a partir de $t=15,0 \mathrm{~s}$ devido a passagem da vazão da descarga vinda da bacia sanitária. Considerando complexidade da superfície livre e a adoção do valor médio das medições experimentais, de um modo geral, pode-se concluir que os resultados da simulação apresentam uma boa concordância em relação aos experimentais.

Figura 5 - Profundidade do escoamento (Caso A, S3).

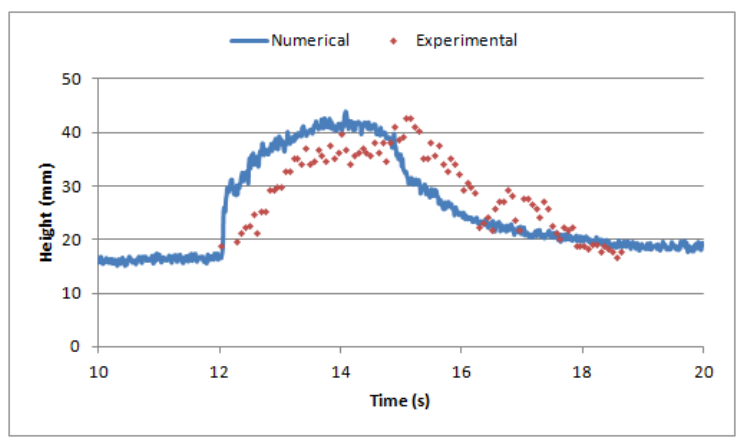

Figura 6 - Vazão (Caso A, S1 a S4).

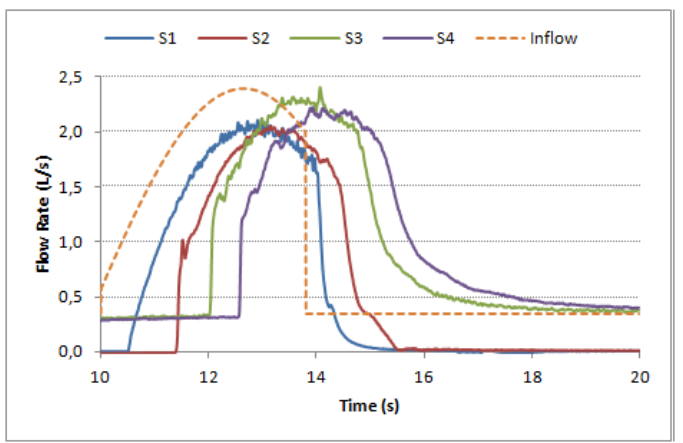

A Figura 6 mostra as vazões computadas nas seções S1, S2, S3 e S4. A vazão total dos efluentes do chuveiro, lavatório e bacia sanitária é representada pela linha pontilhada para servir de referência. Nas seções S1 e S2, à montante da junção, a vazão é inicialmente zero. No entanto, nas seções $S 3$ e S4, à jusante da junção, a vazão inicial é igual a $0,35 \mathrm{~L} / \mathrm{s}$ devido ao escoamento constante do chuveiro e lavatório. A descarga na bacia sanitária ocorreu no $t=10,0 \mathrm{~s}$, e atingiu S1 por volta do $t=10,6 \mathrm{~s}$. A partir daí, a vazão aumenta rapidamente até atingir $2 \mathrm{~L} / \mathrm{s}$, que é a vazão máxima da descarga, para depois decair gradualmente até $t=14,0 \mathrm{~s}$, quando ocorre uma queda brusca da vazão devido a fim da vazão da descarga na seção. O perfil de vazão da S2 apresenta comportamento similar a S1. A frente de onda da descarga alcança S2 no $t=11,4 \mathrm{~s}$. Na $\mathrm{S} 2$ o aumento da vazão é mais brusco. Esta diferença é devida ao efluente do chuveiro e lavatório e acumulado inicialmente à montante da $\mathrm{S} 2$ sendo empurrado pela descarga e cruzando rapidamente $\mathrm{S} 2$. Os perfis de vazão na S3 e S4 são similares ao da S1, exceto 
com uma defasagem de tempo e adicionada a vazão constante de $0,35 \mathrm{~L} / \mathrm{s}$ do chuveiro e lavatório, além de um decaimento da vazão cada vez mais suave nas seções à jusante.

A vazão no tubo de queda também foi determinada na simulação. A fim de facilitar o emprego deste resultado como vazão de entrada do Caso B, uma função aproximada para descrever a vazão foi obtida:

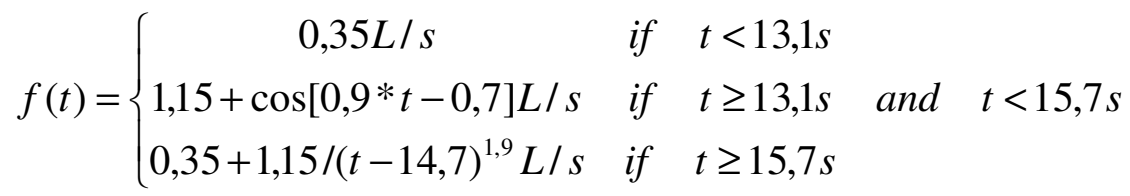

onde, $f=$ vazão na seção de entrada no tubo de queda.

\subsection{Caso B}

Para monitorar a altura do escoamento e a vazão no subcoletor ligado ao tubo de queda por dois joelhos de $45^{\circ}$, foram consideradas cinco seções, S1 a S5, que no Caso B estão localizadas, respectivamente, a $0,25,0,50,0,75,1,00$ e $1,25 \mathrm{~m}$ do ponto inicial do subcoletor horizontal. Vale ressaltar que na simulação do Caso B a vazão de saída do Caso A (Eq. 10) é usada como vazão de entrada no tubo de queda considerando o escoamento anular com espessura de $2,2 \mathrm{~mm}$.

Figura 7 - Profundidade do escoamento (Caso B, S1 a S5).

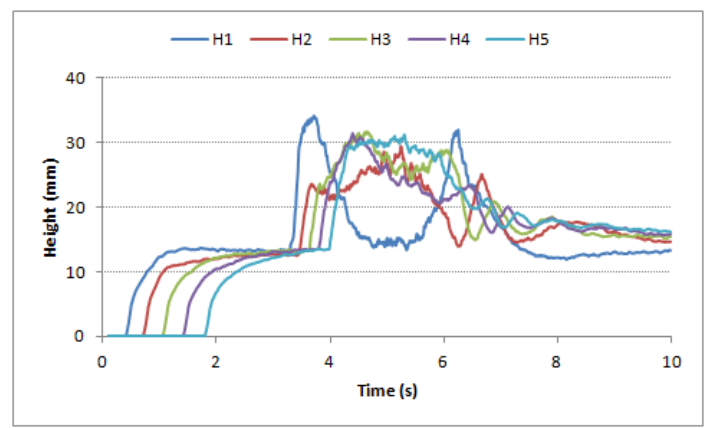

Figura 8 - Vazão (Caso B, S1 a S5).

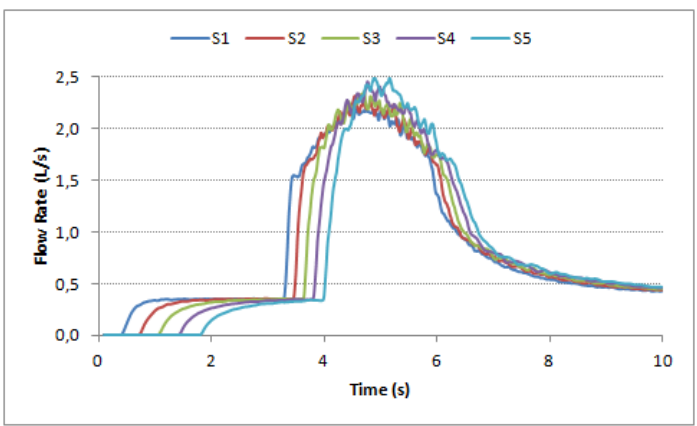

A Figura 7 mostra as séries temporais da altura do escoamento nas seções S1 a S5 obtidas na simulação. Todas as seções apresentam uma elevação inicial devido ao influxo oriundo do chuveiro e lavatório. Nas seções S3, S4 e S5, os comportamentos são similares com uma elevação rápida, mantendo o patamar por mais ou menos 1 segundo e depois o decaimento suave após a passagem da frente de onda devido à descarga da bacia sanitária. No entanto, nas seções $\mathrm{S} 1$ e $\mathrm{S} 2$, e principalmente na $\mathrm{S} 1$, próximo ao joelho, são registrados dois picos: o primeiro quando o fluxo devido à descarga atinge a seção. Este decai rapidamente enquanto a frente de onda atinge as seções subsequentes. Por fim, o segundo pico ocorre quando os níveis nas seções à jusante diminuem, formando uma outra onda após a passagem da frente da onda devido à descarga.

Os perfis de vazão nas seções S1 a S5 são apresentados na Figura 8. Na S1, a vazão inicial de $0,35 \mathrm{~L} / \mathrm{s}$ aumenta abruptamente para $2,3 \mathrm{~L} / \mathrm{s}$ com a chegada da descarga, e reduz para $1,8 \mathrm{~L} / \mathrm{s}$ no $\mathrm{t}=6,0 \mathrm{~s}$ para decair assintoticamente de volta para $0,35 \mathrm{~L} / \mathrm{s}$. De modo geral, as curvas são similares nas 5 seções, o pico de vazão tende a ser um pouco mais elevado e a duração do transiente tende a ser mais curto para seções à jusante. 


\section{CONSIDERAÇÕES FINAIS}

Este trabalho apresenta uma nova abordagem tendo como base o método de partículas para a investigação do complexo escoamento tridimensional e transiente com superfície livre no interior dos sistemas prediais de esgoto sanitário. Como um dos passos iniciais da modelagem para investigar o impacto da redução da vazão no desempenho autolimpante do sistema, o caso de um banheiro localizado no segundo andar foi analisado. Apesar da simplicidade, o caso leva em conta duas singularidades geométricas típicas: ramal de esgoto com junção de $45^{\circ}$ e subcoletor conectado ao tubo de queda por dois joelhos de $45^{\circ}$. Os resultados das simulações mostram boa concordância com as medições experimentais, e informações detalhadas obtidas sobre o escoamento permitiram o entendimento minucioso do processo hidrodinâmico, abrindo assim, a possibilidade de ensaios virtuais para o aprimoramento do sistema. Como trabalho futuro tem-se a execução de simulações com resoluções mais altas, a modelagem da tensão superficial e molhabilidade, e a simulação do processo de transporte de sólidos. Deste modo, o presente trabalho, que resultou do projeto de desenvolvimento do simulador computacional para aplicações genéricas contando com financiamento do setor produtivo, e com aplicação focada no estudo do desempenho dos sistemas prediais de esgoto sanitário contribui tanto na inovação da abordagem como na obtenção dos resultados inéditos que ajudam a esclarecer os mecanismos dos escoamentos nos sistemas prediais de esgotos sanitários.

\section{AGRADECIMENTOS (OPCIONAL)}

À Fundação de Amparo à Pesquisa do Estado de São Paulo (FAPESP), processo $n^{\circ}$ 2014/16362-1, pelo apoio na divulgação da pesquisa.

\section{REFERÊNCIAS}

CHENG C.L., LIAO W.J., HE K.C., HSIEH C.H. and LIU Y.C. Empirical approach to main drain system design and solid transportation performance in buildings., Building Services Engineering Research and Technology, first published on January 4, 2013 as doi: $10.1177 / 0143624412460239$.

CHENG, L. Y.; OLIVEIRA, L. H.; FAVERO, E. H. Particle-based numerical analysis of drainage flow inside building system. In: CIB W62 Symposium on Water Supply and Drainage for Buildings, 2012, Sidney. The 38th International Symposium CIB W062 on Water Supply and Drainage for Buildings. Edinburgh, Scotland. 27-30 Aug. 2012. p.227 - 238.

CHENG, L. Y.; OLIVEIRA, L. H.; FAVERO, E. H. Analysis of building drainage system using particle-based numerical simulation. In: The Proceedings of the Fourteenth International Conference on Civil, Structural and Environmental Engineering Computing. (CC2013), Paper 222, September 3-6, 2013, Cagliari, Italy.

LEE, E. W. Preliminary study on the application of computational fluid dynamics to building drainage system design. Surveying and Building Environment, v. 17, n.1, p.35-44, June, 2006.

JACK L.B. and SWAFFIELD J.A. Embedding sustainability in the design of water supply and drainage systems for buildings., Renewable Energy, v.34, p.2061-2066, 2009.

KOSHIZUKA, S. and OKA Y. Moving-Particle Semi-Implicit method for fragmentation of incompressible fluid., Nuclear Science and Engineering, 123, 421-434, 1996.

McDOUGALL J.A. and SWAFFIELD J.A. The influence of water conservation on drain sizing for building drainage systems., Building Services Engineering Research \& Technology, v. 24, n.4, 2003. 